ORIGINAL ARTICLE

\title{
Insights from the sharp end of intravenous medication errors: implications for infusion pump technology
}

\author{
M Husch, C Sullivan, D Rooney, C Barnard, M Fotis, J Clarke, G Noskin
}

Qual Saf Health Care 2005;14:80-86. doi: 10.1136/qshc.2004.011957

See end of article for authors' affiliations

\section{Correspondence to:} M Husch, Research Pharmacist Coordinator Patient Safety Team Northwestern Memorial Hospital, 676 North St Claire, Chicago, IL 60611,

USA; mhusch@nmh.org

Accepted for publication 21 January 2005
Background: Intravenous (IV) medication errors are a common type of error identified in hospitals and can lead to considerable harm. Over the past 20 years there have been several hundred FDA reported incidents involving IV pumps, many of which have led to patient deaths.

Objective: To determine the actual types, frequency, and severity of medication errors associated with IV pumps. To evaluate the likelihood that smart pump technology without an interface to other systems could have prevented errors.

Methods: Using a point prevalence approach, investigators prospectively compared the medication, dose, and infusion rate on the IV pump with the prescribed medication, doses, and rate in the medical record. Preventability with smart pump technology was retrospectively determined based on a rigorous definition of currently available technology.

Results: A total of 426 medications were observed infusing through an IV pump. Of these, 285 (66.9\%) had one or more errors associated with their administration. There were 389 documented errors overall; 37 were "rate deviation" errors and three of these were judged to be due to a programming mistake. Most of the documented events would not have caused patient harm (NCC MERP category C). Only one error would have been prevented by smart pump technology without additional interface and software capabilities.

Conclusion: Medication errors associated with IV pumps occur frequently, have the potential to cause harm, and are epidemiologically diverse. Smart pumps are a necessary component of a comprehensive safe medication system. However, currently available smart pumps will fail to generate meaningful improvements in patient safety until they can be interfaced with other systems such as the electronic medical record, computerized prescriber order entry, bar coded medication administration systems, and pharmacy information systems. Future research should focus on the effectiveness of new technology in preventing latent and active errors, and on new types of error that any technology can introduce.
(1) ntravenous (IV) medication errors have led to considerable patient harm ${ }^{1}$ and are abundant. ${ }^{2}$ Several reports estimate that medication errors occurring in the administration phase of the medication use process are now the most frequent type of mistake occurring in hospitals. ${ }^{3-5}$ They are also the most dangerous type of medication error as they are least likely to be intercepted ${ }^{6}$ and often involve medications with a narrow therapeutic range (such as "high alert medications"). ${ }^{7}$ Since 1985 there have been several hundred reports of incidents involving infusion pumps reported to the Federal Drug Administration (FDA) and many of these have led to patient deaths. ${ }^{8}$ The scope of this problem is daunting since an estimated $90 \%$ of hospitalized patients receive medication via the IV route, ${ }^{9}$ and infusion pumps are used to deliver most IV continuous infusion and intermittent medications in the USA. ${ }^{10}$

Several studies of the epidemiology of IV medication errors have been performed but have not been well quantified due to varying definitions and methodologies. ${ }^{12611} 12$ Data derived from hospital based incident reporting systems have been used to evaluate IV pump errors. However, the results are unreliable and not well quantified due to underreporting. ${ }^{13-15}$ To our knowledge, there has been no independent prospective study conducted that offers detailed analysis of the types, frequency, severity and causes of IV administration errors specifically associated with IV pumps.

Manufacturers have claimed that "smart pumps" can avert one potentially life threatening error every 2.6 days at an average 350-bed hospital. ${ }^{16}$ Smart pumps are described in box 1. Smart pump technology currently does not have the capability to interface with other information technologies (IT) such as computerized prescriber order entry (CPOE) and bar coded medication administration systems (BCMA). ${ }^{18}$ To our knowledge there has been no independent systematic study of the likelihood that smart pump technology would prevent errors in IV pump usage observed through a rigorous study.

A multidisciplinary team comprised of nurses, pharmacists, and biomedical engineers performed a failure mode and effects analysis (FMEA) to identify potential failure modes or system vulnerabilities associated with IV pumps at a major academic medical center. The FMEA was initially conducted as part of a hospital-wide quality improvement project. Many possible failure modes were identified by the team including programming errors, patient monitoring errors, and device errors. However, to improve the safety of IV pump utilization, we needed a better understanding of the actual extent and nature of these errors. The FMEA team acknowledged the inadequacies of our voluntary incident reporting system and therefore its inability to provide us with this information. The team performed an observational, prospective investigation using a point prevalence approach.

The objectives of this investigation were to determine the type, frequency, and severity of errors associated with IV infusion pumps. We also estimated the likelihood that smart

Abbreviations: $B C M A$, bar coded medication administration; $C P O E$, computerized prescriber order entry; EMR, electronic medical record; PIS, pharmacy information system 
Box 1 Smart pumps (dose error reduction systems) ${ }^{17}$

- Intravenous pumps that have software that checks programmed doses against preset limits specific to a drug and clinical location.

- The clinician may either override an alert (soft limit) or not be allowed to continue at all (hard limit), depending on preset limits.

pump technology could have prevented each "rate deviation" error.

\section{METHODS}

This study took place at a 725-bed tertiary care academic medical center in Chicago on a historically high volume day of the week (Thursday) in January 2003. At the time of the study, medications orders were handwritten on multicopy blank order forms or preprinted order forms that required partial completion and signature. According to institutional policy, all telephone and verbal orders are required to be reduced to writing in the patient's medical record by nurses or other clinicians-that is, medical students and pharmacists-and countersigned. Except in urgent situations, a pharmacist reviewed all medication orders and entered them into the pharmacy computer system before dispensing. With the exception of standard IV fluids - that is, I liter bags of premixed dextrose $5 \%$ in water (D5\%W), $0.9 \%$ normal saline (NS) - which are nursing unit floor stocked items, all continuous infusion IV medications and intermittent IV medications that are infused via an IV pump are prepared in the pharmacy and dispensed to the nursing units for administration.

Data were collected for one day from 08.00 hours to 17.00 hours. All epidural, patient controlled analgesia (PCA) and general use IV infusion pumps in use on inpatient care units were included in the investigation. Excluded from the study were pumps in use in the operating rooms, recovery rooms, labor and delivery units, post-partum units, and the emergency department due to low IV pump utilization or high patient turnover. To avoid observer induced bias, ${ }^{19}$ the nursing staff was not aware that this investigation was taking place. The Northwestern University institutional review board (IRB) approved the study.

Four teams were created, each consisting of a pharmacist and nurse from the Patient Safety Research and Demonstration Team at our institution. On assigned nursing units, using a prospective point prevalence approach, each team compared the infusing medication, dose, and infusion rate on the pump with the prescribed medication, dose, and rate in the medical record. All orders were obtained from handwritten paper-based medical records and all IV fluids were considered medications. Documentation of the ordered rate on the label of the infusing medication was also assessed. Presence of a correct patient identification (ID) band was recorded for each patient. All data were collected on a standardized form to which each team had been formally oriented before the study.

\section{Determination of errors}

An error was defined as any preventable event that may cause or lead to inappropriate IV medication use via an IV pump or patient harm while the medication is in the control of the healthcare professional, patient, or consumer. Such events may be related to professional practice, healthcare products, procedures and systems including order communication, product labeling, compounding; dispensing; administration; education; monitoring; and use. ${ }^{20}$ This definition, although slightly modified for the methodology of this study, was established in 1995 by the National Coordinating Council for Medication Error Reporting and Prevention (NCC MERP). ${ }^{20}$ It includes deviations in the administration of a drug from the physician's prescription or from our hospital's policies. All deviations were identified as errors even if they only had the capacity to contribute to patient harm; examples would include a missing identification bracelet or lack of a documented rate on a medication label. More than one error could be documented for a single observed IV medication. For example, a patient without an ID band could also have a medication infusing at a different rate from the rate in the physician order. In this case, two errors would be documented for this observation, both a "patient identification" error and a "rate deviation" error.

All the investigators who participated in data collection were trained regarding the various types of errors and their proficiency was documented (box 2). In order to confirm that an error was present, both investigators had to agree that an error was made. When an error was discovered and agreed upon, the staff nurse caring for that particular patient was discreetly informed so that it could be corrected as warranted.

\section{Determination of potential severity}

The potential severity for each error was determined. Each error was classified into one of nine letter designated categories according to the NCC MERP index for categorizing medication errors. ${ }^{20}$ This taxonomy was slightly modified as described in a previously published investigation. ${ }^{21}$ These categories, in ascending order of severity, include capacity to cause error (A), an error occurred but did not reach the patient (B), errors unlikely to cause harm despite reaching the patient $(C)$, errors that would have required increased monitoring to preclude harm (D), errors likely to cause temporary harm (E), errors that would have caused

\section{Box 2 Definitions of error types}

- Rate deviation: a different rate is displayed on the pump from that prescribed in the medical record. Also refers to weight based doses calculated incorrectly including using a wrong weight.

- Incorrect medication: a different fluid/medication as documented on the IV bag label is being infused compared with the order in the medical record.

- Delay of rate or medication/fluid change: an order to change medication or rate not carried out within 4 hours of the written order per institution policy.

- No rate documented on label: applies both to items sent from the pharmacy and floor stocked items per institution policy.

- Incorrect rate on label: rate documented on the medication label is different from that programmed into the pump. Applies both to items sent from the pharmacy and floor stocked items.

- Patient identification (ID) error: patient either has no ID band on wrist or information on the ID band is incorrect.

- Unauthorized medication: fluids/medications are being administered but no order is present in medical record. This includes failure to document a verbal order. 
temporary harm and prolonged hospitalization $(\mathrm{F})$, errors which would have produced permanent harm $(G)$, errors that would have been life threatening $(\mathrm{H})$, and errors that would likely have resulted in death (I). The assigned severity rating was based on the potential for the error to have resulted in patient harm if it had not been intercepted.

Errors such as "no documented rate on medication label" or "missing patient identification bands" were assigned a rating of " $\mathrm{C}$ " (an error occurred that reached the patient but did not cause harm). All others were rated based on the professional judgment and consensus of the nurse and pharmacist investigators. If the team members could not agree on a rating, a third investigator from one of the other teams reviewed the event to determine the appropriate category. If necessary, further investigation into the cause of the error took place via an interview with the nurse and a more in-depth chart review to determine most accurately the potential severity of the observed error.

At the conclusion of the study all errors assigned a severity rating of " $\mathrm{D}$ " or greater were independently reviewed by one of two board certified internists. This was accomplished by retrospective chart review and these individuals were blinded to the original ratings. Final assignment of severity rating was determined only after consensus among physicians and the original evaluators.

\section{Evaluating the cause of rate deviation errors and the potential impact of smart pump technology}

Three investigators (two nurses and a pharmacist) retrospectively rated all "rate deviation" errors as to whether or not they were caused by a programming mistake and whether smart pump technology would have prevented the error (yes or no). Only the "rate deviation" errors were individually analyzed for smart pump preventability as it is already recognized that no other types of errors (such as unauthorized medication errors, patient identification errors, no rate on label errors, and wrong medication errors) would be prevented by smart pump technology without an interface with other systems. ${ }^{22}$

All three investigators had to agree on the rating. Ratings regarding the likelihood that an error was due to a programming mistake were based on over 10 years of clinical experience each and notes taken by the investigators at the time of data collection. Ratings about the effectiveness of smart pump technology were based on an extensive review of the published literature, clinical experience, and personal communication with clinicians and manufacturers who are experienced with this technology.

\section{Evaluating errors identified via traditional incident reporting versus trained observers}

To assess our hypothesis that data generated via our current voluntary incident reporting system is unreliable and sporadic, we compared the data from this study with data generated via the incident reporting system. Our incident reporting system is paper based, voluntary, and can be anonymous. Additionally, as only "rate deviation" and "wrong medication" errors in relation to IV pumps are typically reported via our institutional incident reporting system, we had to limit our comparison of data generated in this study with data generated via incident reports to these two types of error.

\section{RESULTS}

\section{Frequency, type and potential severity}

During the data collection period 486 inpatients were included in the study. An IV pump was used for 286 $(58.8 \%)$ of these patients during the data collection period and 426 medications were observed infusing through these pumps. Of the 426 medications observed, 285 (66.9\%) had one or more errors associated with their administration. There were 389 errors overall; 37 were "rate deviation" errors and three of these were judged to be due to a programming mistake.

Regarding potential harm, most of the documented errors fell into NCC MERP category " $\mathrm{C}$ ". The number, frequency, and potential severity of these errors are shown in table 1 . Examples of some of the errors that were rated with a potential severity greater than " $\mathrm{D}$ " are shown in table 2 .

\section{Smart pump projected efficacy in error prevention}

Of the 37 deviation errors, $36(97.3 \%)$ were rated unlikely to be prevented by smart pump technology. The ratings were based on the assumption that the clinicians would have used the smart pump technology to its fullest capacity. Only one of the errors was rated as likely to have been prevented by current smart pump technology. This error involved heparin which was infused at a rate of $2 \mathrm{ml} / \mathrm{h}$ (200 units/h) compared with the ordered rate of $13 \mathrm{ml} / \mathrm{h}$ (1300 units/h) for the treatment of a venous thromboembolism. It is expected that this dose would be outside the normal dose range and therefore would be queried by smart pump software. All other rate deviation errors were related to reasons other than programming (which would make them not susceptible to prevention with smart pump technology), or were errors not significant enough to have been detected by smart pump technology because the mis-programmed doses would not have exceeded routine dose limits.

\section{Errors identified by traditional incident reporting versus trained observers}

There were 48 incident reports documenting data on "rate deviation" and "wrong medication" errors over a 2 year period at our institution. However, we observed 55 "rate deviation" and "wrong medication" errors in a 9 hour period. As previously documented in the literature, voluntary paperbased incident reporting systems are unreliable for collecting

Table 1 Number, frequency, and potential severity of each type of error

\begin{tabular}{|c|c|c|c|c|c|c|}
\hline \multirow[b]{2}{*}{ Type of error } & \multirow[b]{2}{*}{ Total $(n=389)$} & \multirow{2}{*}{$\begin{array}{l}\text { Frequency per medication } \\
\text { observations* } \\
\text { ( } n=426 \text { ) }\end{array}$} & \multicolumn{4}{|c|}{ NCC MERP severity rating } \\
\hline & & & C & D & $\mathbf{E}$ & $\mathbf{F}$ \\
\hline No rate on label & 195 & $46 \%$ & 195 & & & \\
\hline Unauthorized medication & 68 & $16 \%$ & 65 & & 3 & \\
\hline Patient identification error & 55 & $13 \%$ & 55 & & & \\
\hline Rate deviation & 37 & $9 \%$ & 29 & 4 & 1 & 3 \\
\hline Incorrect rate on label & 16 & $4 \%$ & 16 & & & \\
\hline Incorrect medication & 14 & $3 \%$ & 11 & 2 & 1 & \\
\hline Delay of rate or medication change & 4 & $1 \%$ & 2 & 2 & & \\
\hline Total & & & $373(96 \%)$ & $8(2 \%)$ & $5(1 \%)$ & $3(1 \%)$ \\
\hline
\end{tabular}




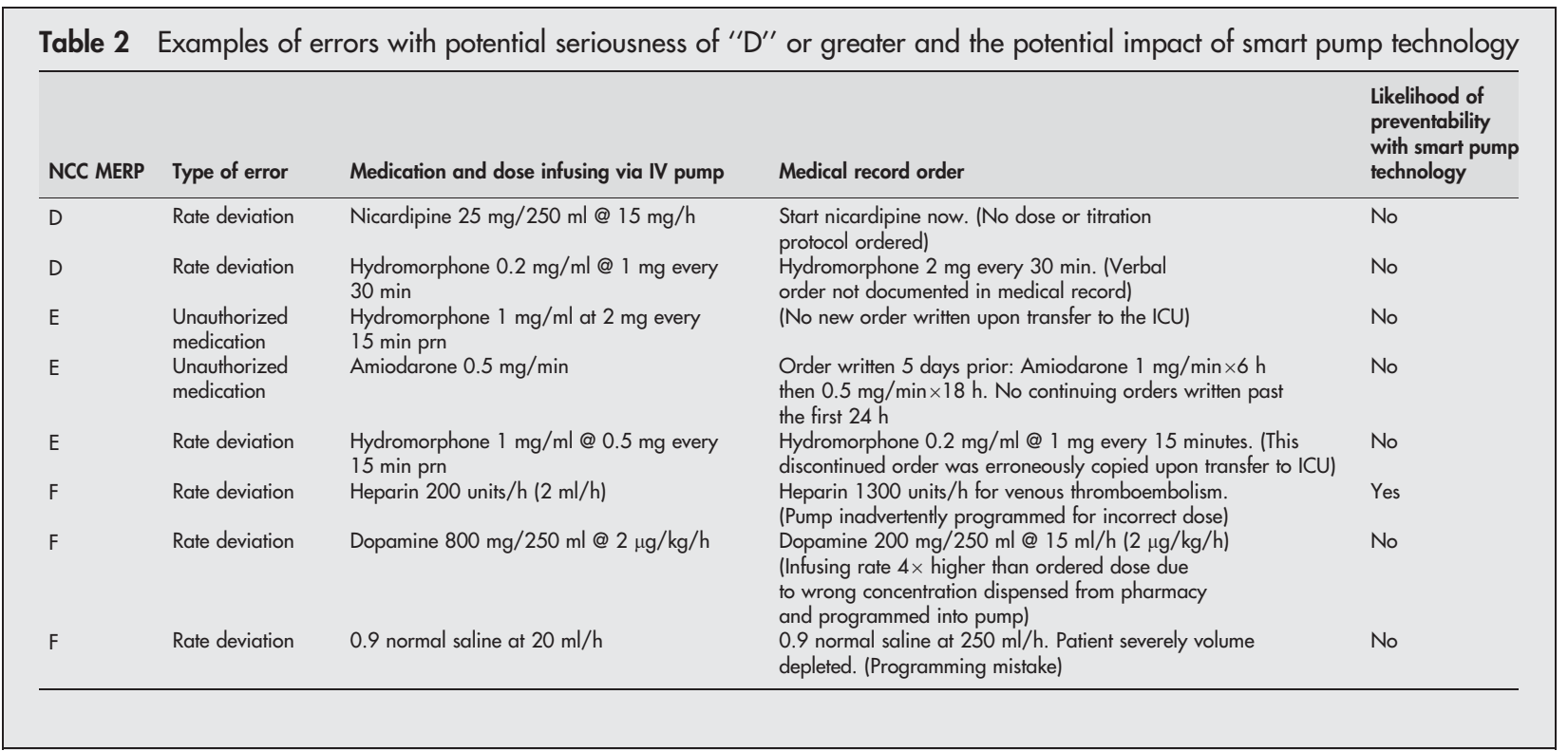

and analyzing medication errors, particularly those which do not cause immediate and observable harm. ${ }^{13-15}$

\section{DISCUSSION}

This study provides evidence that IV medication errors associated with IV pumps are common but are more epidemiologically diverse than expected. Specifically, rate deviation errors as a result of a programming mistake were less frequent than expected, while errors associated with orders, documentation, labeling and patient identification were more frequent. This evidence gives impetus for healthcare organizations and IV pump vendors to appreciate that latent errors and circumstances can have at least as much impact on patient harm as active failures, and include these types of errors in the analysis of safer systems design.

Some investigators examining errors associated with IV pumps assume that the definition of an IV pump error is a programming error. ${ }^{23}$ By failing to include other types of errors associated with IV pump utilization, the magnitude and root cause of the problems would be significantly underestimated. ${ }^{24}$ This could lead to a perception that IV medication errors associated with an IV pump can be mitigated (with "smart" pump technology) but, instead, allow undetected weaknesses in the medication use system to persist, resulting in patient harm. Tamuz and colleagues concluded that the definitions and classifications of medication errors could profoundly enhance an institution's capacity to learn from these events and therefore decrease patient harm..$^{25}$ Because of this, we chose a broad definition for IV pump administration errors in an attempt to gain further insights into the nature of these errors and to understand them better. Expanding the definition of an IV pump administration error beyond merely programming errors to include deviations from policies permitted us to identify events that had the capacity to contribute to patient harm. The profound effects of these latent conditions (such as a missing ID bracelet or a mislabeled IV bag) have been previously described. ${ }^{26}$

The additional data derived from a more inclusive definition will facilitate effective implementation of improved technology. Since almost half the labels on infusing medications either had no current rate documented or contained a different rate than that ordered in the medical record, pumps with bar code scanners that can automatically program pump settings from a machine readable label would be an important technological feature. ${ }^{17} 22$ This technology prevents the pump from activating if there is no rate embedded into the label. Identifying potential system issues before implementation will facilitate introduction of these pumps and potentially prevent new errors.

We identified that a "slip-of-the-finger" type of error such as programming $600 \mathrm{ml} / \mathrm{h}$ instead of $60 \mathrm{ml} / \mathrm{h}$ occurred in only three instances in this study, suggesting more diversity than previously reported..$^{23}$ For example, recurrent causes of "rate deviation" errors include failure to document verbal orders and failure to transcribe the most current order upon transfer to a different unit. A patient who had a current order for hydromorphone $1 \mathrm{mg}$ every $15 \mathrm{~min}$ via a PCA was actually receiving hydromorphone $0.5 \mathrm{mg}$ every $15 \mathrm{~min}$. Upon further investigation it was discovered that, when the patient was transferred to the intensive care unit, previous discontinued orders were inadvertently copied instead of the most recent orders. In this particular instance the patient's actual medication dose was accurate but the paper order was incorrect. If this error had continued indefinitely, any dose change that would be based on the paper record that described a dose that was twice that of what the patient was actually receiving could lead to subsequent under- or overdosing. In another case a patient was receiving amiodarone $0.5 \mathrm{mg} / \mathrm{min}$ via continuous infusion. On the fifth day of treatment it was discovered that there were no orders in the medical record to continue this medication beyond 24 hours. Upon further investigation it was determined that she was ultimately receiving the right medication at the right dose and that the lack of a written order in her medical record was an oversight. If prescribers and other clinicians are unaware of a patient's current medication regimen, serious drug interactions and further errors are not only possible but likely.

Both of these errors are, of course, not intrinsically errors in the IV medication administration node. They are errors in the process of receiving, reconciling, and documenting orders. The important observation here is that the correct administration of IV medications is dependent on a reliable process for taking, verifying, and documenting orders. Any analysis of the safety of the IV medication system must take this into account. Smart pump technology in its current form does not interface with medication orders and is therefore vulnerable to the same failures. 
We found that programming mistakes were infrequent but had the potential to cause harm, and that errors originating in other phases of the medication use process-such as patient identification, unauthorized medication and documentation errors-may have similar potential to cause patient harm. We determined that only one of the 389 documented errors would have been prevented by basic smart pump technology. The intelligence of smart pumps without an interface is limited solely to preventing errors that are the result of incorrect programming, and even this capability is limited to errors that exceed routine dose limits. They do not have the capacity to alert the clinicians to errors involving a wrong dose (which does not match the order), a wrong medication, wrong patient, or wrong indication. ${ }^{22}$ These pumps are not able to detect a change in the patient's condition, intended transfer orders, or an unintended change in the concentration of a refilled continuous drip. For example, an error that involved a dopamine drip that was infusing at four times the prescribed amount was not due to a programming error but rather a dispensing error in which the concentration that was dispensed for a subsequent bag of dopamine was quadruple that in the previously dispensed bag. Upon further investigation it was determined that the error occurred as a result of the way the pharmacy enters into its own computer system those medications that are typically titrated based on clinical response. In fact, the limited safety features of smart pump technology without interface to bar code scanners may not be useful at all as there is no way to require clinicians to use them so they can readily be bypassed. ${ }^{27} 28$

Nevertheless, smart pumps are an important component in a comprehensive safe medication system. Those errors, which they do have the potential to prevent, can be significantly harmful. The one error that may have been prevented was a "rate deviation" error resulting in inadequate anticoagulation with heparin that could have resulted in a life threatening venous thromboembolism.

ECRI (formerly the Emergency Care Research Institute), a leading technology evaluation organization, is now rating pumps without dose error reduction software (smart pumps) as unacceptable. ${ }^{17}$ Other institutions have recently considered the potential impact of smart infusion devices by evaluating alert logs that were downloaded from the quality event trackers incorporated into the software of each pump. ${ }^{29}{ }^{30}$ The results of these investigations were consistent with the results of the present study. The number of times a pump was reprogrammed secondary to an alert was small compared with the number of doses administered; however, the outcomes had the potential to be significant. It should be noted that these studies did not explicitly address the question of whether the "smart" technology was responsible for the identification of these errors, or whether they might have been caught with the prior generation of pumps and conventional practice. It is difficult to draw conclusions regarding the impact of smart pumps on patient outcomes without such an analysis because wrong programming is frequently intercepted by traditional means such as overlapping vigilance, experience, monitoring, and double checks. Without taking this into consideration, reports such as these may overestimate the risk of errors and harm with conventional pumps and practice.

In order to achieve meaningful improvements in patient safety and maximum protection against all IV administration errors, there must be seamless multi-directional communication among smart pumps and (a) an electronic medical record (EMR); (b) a CPOE system with sophisticated rules and alerts and solid human factors engineering; (c) a BCMA system that assures correct patient/drug/medication/dose/ route/schedule per the original order; and (d) the pharmacy system to allow pharmacists to verify orders, dispense accurately, monitor infusion rates and volumes, and to better anticipate the needs of the patients. All nodes of the medication use process from prescribing through administration and monitoring should be able to communicate electronically with one another in real time (table 3 ).

With the addition of any new technology we must also be wary of the introduction of new types of error. ${ }^{31}$ This potential has been demonstrated with other technology such as CPOE. Bates and coworkers reported an initial increase in potassium chloride dosing errors after implementation of CPOE with clinical decision support. ${ }^{32}$ Although little research has been done to quantify new types of errors

Table 3 Conceptual model of technology support for a complete medication safety system for IV medication administration

\begin{tabular}{|c|c|c|c|}
\hline Node & Order & Transcribe and dispense & Administer and monitor \\
\hline Features & $\begin{array}{l}\text { CPOE with decision support receives and } \\
\text { supports with logic accurate appropriate } \\
\text { order, dosing ranges, interactions, allergies } \\
\text { Communicates order to PIS }\end{array}$ & $\begin{array}{l}\text { Pharmacy system receives electronically } \\
\text { Profiled order communicates to EMR } \\
\text { and populates the eMAR for later charting } \\
\text { of administration } \\
\text { Dispensed medication carries bar coded label } \\
\text { with patient name, drug, dose, rate, } \\
\text { route, start time, interval }\end{array}$ & $\begin{array}{l}\text { Dispensed medication is read by a bar code } \\
\text { scanner (BCMA) which results in automatic } \\
\text { programming of the infusion pump } \\
\text { Bar code reader requires input in full } \\
\text { agreement from three sources: EMR (the order), } \\
\text { patient wristband (patient ID), and the } \\
\text { medication in the pump } \\
\text { "Smart" pump discerns doses programmed } \\
\text { outside preset limits specific to a drug and } \\
\text { clinical location* } \\
\text { Pump communicates information on } \\
\text { administered medication back to eMAR } \\
\text { EMR may automatically generate related } \\
\text { nursing tasks, e.g. vital signs at intervals } \\
\text { appropriate to the medication, or orders such } \\
\text { as follow up lab work }\end{array}$ \\
\hline Examples & $\begin{array}{l}\text { ch can be prevented } \\
\text { Prescribing errors } \\
\text { Lost orders } \\
\text { Undocumented verbal orders }\end{array}$ & $\begin{array}{l}\text { Transcription errors } \\
\text { Prescribing errors which missed first screen } \\
\text { Delay of medication administration due to failure } \\
\text { of nurse to notice new orders }\end{array}$ & $\begin{array}{l}\text { Wrong patient or no patient ID band } \\
\text { Infusion pump programming errors } \\
\text { Prescribing errors if any have missed earlier } \\
\text { screens } \\
\text { Wrong or missing label on dispensed } \\
\text { medication } \\
\text { Omitting/forgetting to document administered } \\
\text { medication } \\
\text { Omitting/forgetting post-medication } \\
\text { monitoring and labs }\end{array}$ \\
\hline
\end{tabular}

CPOE, computerized prescriber order entry; PIS, pharmacy information system; EMR, electronic medical record; eMAR, electronic medication administration record. *Without interface to other systems, currently available smart pumps are limited to this feature. 
possible with smart pumps, they must be considered. Because complex drug libraries have many selections, introducing the possibility of selecting the wrong item in a lengthy list, one institution decided they posed a bigger problem than having no library at all and therefore removed most of them. ${ }^{33}$ Additionally, errors in the drug library itself could affect many patients before being caught and corrected. Ongoing research should focus on new types of errors that this technology could introduce.

To facilitate comparisons with other studies we also determined the number of errors that were initiated in the "sharp end" of the medication use process compared with the "blunt end". Active or "sharp end" errors are defined as errors that occur at the front end of the system (in this case, administration errors) and latent or "blunt end" errors are defined as errors or conditions which can lead to an adverse effect or create an accident opportunity. ${ }^{26}$ Of the 389 observed errors, $119(31 \%)$ were considered sharp end errors initiated in the administration phase of the medication use process. For example, a volume depleted oncology patient who had an order to receive $250 \mathrm{ml} / \mathrm{h}$ normal saline was actually receiving $20 \mathrm{ml} / \mathrm{h}$ normal saline. Upon further investigation it was determined that the ordered rate was correct and that the lower rate the patient was actually receiving was due to a programming error. Another example of a sharp end error involves the delay of carrying out an order to discontinue IV fluids for a patient determined to be fluid overloaded. Upon further investigation of this error it was determined that the patient required a dose of an IV diuretic partially due to this delay. The rate of sharp end errors (administration errors) documented in this investigation is comparable to a previous publication where $34 \%$ of their errors occurred in the administration phase. ${ }^{6}$

There are several limitations to our study. It was conducted at a large academic medical center where the amount of interdisciplinary clinician support and education may be different from that at other institutions, thereby limiting its generalizability. However, other IV administration error studies performed at large academic medical centers are widely applicable. ${ }^{34}$ Also, a study of adverse events that involved infusion pumps collected over a 10 year period by the FDA concluded that there were no differences in the types of adverse events reported by hospitals compared with reports from home health agencies. ${ }^{8}$ Another limitation of this investigation is that we performed a point prevalence study. It is possible that the number of IV administration pump errors that occurred on the day that data was collected was different from other days. Thursday, a high census day, was chosen as the best day to perform this study in order to collect as much meaningful data as possible.

\section{Key messages}

- Medication errors associated with IV pumps are common and have the potential to cause harm.

- Errors occurring in phases other than the administration node of the medication use process can result in harm associated with IV pump utilization.

- Smart pumps with dose error reduction systems will fail to generate meaningful improvements in patient safety unless they are fully integrated with information systems such as electronic medical record (EMR), computerized prescriber order entry (CPOE), bar coded medication administration (BCMA), and the pharmacy information system (PIS).
Because most of the errors were intercepted by research team members, the potential harm of the error if it had continued indefinitely was estimated based on our best clinical judgment. Studies have shown that judgments about adverse events due to medical care have poor to moderate inter-rater reliability. ${ }^{35}$ However, all of the researchers have worked together on similar studies where inter-rater reliability was high. ${ }^{21}$ In addition, all errors determined to have a severity rating of " $\mathrm{D}$ " or greater were retrospectively reviewed by two board certified internists to confirm potential severity assignment. The likelihood that smart pump technology would have prevented an observed error was based on our current knowledge of how this technology functions. Our knowledge about the benefits and limitations of this technology will continue to evolve as more information becomes available.

In summary, IV medication errors associated with infusion pumps occur frequently, have the potential to cause harm, and are epidemiologically diverse. The extent and nature of these errors remains mostly unknown due to the lack of research. Pumps equipped with software that checks programmed doses against preset limits specific to a drug and clinical location can decrease the likelihood of a small number of these medication errors. However, based on this study and others, it appears that these dose error reduction systems will fail to generate meaningful improvements in patient safety unless they are interfaced with other medication use information systems such as an EMR, CPOE, BCMA, and PIS. Future research should focus both on the effectiveness of new technology in preventing both latent and active errors as well as new types of error that any technology can introduce.

\section{Authors' affiliations}

M Husch, C Sullivan, D Rooney, C Barnard, M Fotis, J Clarke, G Noskin, Northwestern Memorial Hospital, Feinberg School of Medicine, Northwestern University, Chicago, USA

Supported by the State of Illinois, Department of Public Aid, and Northwestern Memorial Hospital.

The authors have no competing interests with the material presented in this manuscript.

\section{REFERENCES}

1 Taxis K, Barber N. Ethnographic study of incidence and severity of intravenous drug errors. BMJ 2003;326:684-7.

2 Kaushal R, Bates DW, Landrigan C, et al. Medication errors and adverse drug events in pediatric inpatients. JAMA 2001;285:2114-20.

3 Runy L. The cause and effect of medication errors. Hosp Health Netw April 2004;30..

4 Anon. Summary of the 1999 information submitted to MEDMARX. A national database for hospital medication error reporting. Rockville, MD: United States Pharmacopeia, 2000.

5 Hicks RW, Cousins DD, Williams RL. Summary of information submitted to MEDMARX in the year 2002: the quest for quality. Rockville, MD: United States Pharmacopeia, 2003.

6 Bates DW, Cullen DJ, Laird N, et al. Incidence of adverse drug events and potential adverse drug events: implications for prevention. JAMA 1995;274:29-34.

7 Cohen MR, Kilo CM. High-alert medications: safeguarding against errors. In: Cohen MR, ed. Medication errors. Washington, DC: American Pharmaceutical Association, 1999:5.1-5.40.

8 Brown LS, Morrison AE, Parmentier CM, et al. Infusion pump adverse events: experience from medical device reports. J Intraven Nurs 1997;20:41-9.

9 Baranowski L. Presidential address: take ownership. J Intraven Nurs 1995; 18:162-4.

10 Flynn F, Mohr L, Lawlor-Klean P. Right programming of pumps to prevent errors in the infusion process. Jt Comm J Qual Saf 2003;29:37-40.

11 Herout PM, Erstad BL. Medication errors involving continuously infused medications in a surgical intensive care unit. Crit Care Med 2004;32:428-32.

12 Calabrese AD, Erstad BL, Brandl K, et al. Medication administration errors in adult patients in the ICU. Intensive Care Med 2001;27:1592-8.

13 O'Neil AC, Peterson LA, Cook EF, et al. Physician reporting compared with medical record review to identify adverse medical events. Ann Intern Med 1993; 1 19:370-6. 
14 Bates DW, Evans RS, Murff $\mathrm{H}$, et al. Detecting adverse events using information technology. J Am Med Inform Assoc 2003;10:115-28.

15 Stanhope N, Crowley-Murphy M, Vincent C, et al. An evaluation of adverse incident reporting. J Eval Clin Pract 1999;5:5-12.

16 Rosenthal K. Smart pumps help crack the safety code. Nurs Manage 2004;35:49-51.

17 Emergency Care Research Institute. General-purpose infusion pumps (evaluation). Health Devices 2002;31:353-87.

18 Emergency Care Research Institute. Bar-coded medication administration (BCMA) systems (evaluation). Health Devices 2003;32:373-81.

19 Barker KN. Data collection techniques: observation. Am J Hosp Pharm 1980;37:1235-43.

20 National Coordinating Council for Medication Error Reporting. Prevention (NCC MERP). NCC MERP index for categorizing medication errors. Available at http://www.nccmerp.org (accessed 20 January 2003)

21 Bobb A, Gleason K, Husch M, et al. The epidemiology of prescribing errors: the potential impact of Computerized Prescriber Order Entry. Arch Intern Med 2004; 164:785-92.

22 Emergency Care Research Institute. General-purpose infusion pumps: Evaluating the B. Braun Outlook Safety Infusion System (evaluation). Health Devices 2003;32:382-94

23 Eskew JA, Jacobi J, Buss WF, et al. Using innovative technologies to set new safety standards for the infusion of intravenous medications. Hosp Pharm 2002;37:1179-89.

24 McNutt RA, Abrams R, Aron DC, et al. Patient safety efforts should focus on medical errors. JAMA 2002;287:1997-2001.
25 Tamuz M, Thomas EJ, Franchois KE. Defining and classifying medical error: lessons for patient safety reporting systems. Qual Saf Health Care 2004; 13:13-20.

26 Reason J. Human error: models and management. BMJ 2000;320:768-70.

27 Eskew JA. Using intravenous medication technology at the point of care. Proceedings of the 38th Annual ASHP Midyear Clinical Meeting, New Orleans, Lovisiana, December, 2003.

28 Robert T, Rough S. Evaluating the impact of smart pump technology on medication error rates. Poster presentation at the 38th Annual American Society of Health-Systems Pharmacists (ASHP) Midyear Clinical Meeting, New Orleans, Lovisiana, December, 2003

29 Malashock CM, Shull S, Gould A. Effect of smart infusion pumps on medication errors related to infusion device programming. Hosp Pharm 2004:39:460-9.

30 Wilson K, Sullivan M. Preventing medication errors with smart infusion technology. Am J Health Syst Pharm 2004;61:177-83.

31 Ash JS. Unintended consequences of information technology. J Am Med Inform Assoc 2004;11:104-12.

32 Bates DW, Teich JM, Lee J. The impact of computerized physician order entry on medication error prevention. J Am Med Inform Assoc 1999:6:313-21.

33 Tourville J. Automation and error reduction: IV pump errors. Available at http://www.uspharmacist.com (accessed 17 May 2004).

34 Taxis K, Barber N. Causes of intravenous medication errors: an ethnographic study. Qual Saf Health Care 2003;12:343-8.

35 Thomas EJ, Lipsitz SR, Brennan TA. The reliability of medical record review for estimating adverse event rates. Ann Intern Med 2002;136:812-6. 